\title{
Correspondence
}

\section{Dendritic Cell Maturity and Obstructive Airway Disease}

\section{To the Editor:}

We read with interest the article by Demedts and colleagues, who evaluated, by immunohistochemistry, the increased infiltration of small airways by dendritic cells (DCs) in chronic obstructive pulmonary disease (COPD) (1). In asthma, we have reported a decrease in the number of mature DCs (mDCs) in endobronchial biopsies of current smokers by comparison with never-smokers (2). Also, we have unpublished data showing that the bronchial mucosa of patients with COPD has fewer mDCs than normal healthy nonsmoker controls and that current smokers have significantly fewer sputum mDCs than ex-smokers with or without COPD. What might be a possible explanation for these apparent differences?

The identification of the distinct immunophenotypes of DCs depends on the method applied. Fluorescence activated cell sorter (FACS) analysis of cells isolated from lung digests, bronchoalveolar lavage fluid, or sputum allows identification of Langerhans' cells (LCs), myeloid DCs types 1 and 2, and plasmacytoid DCs, and distinguishes between mDCs and immature DCs (iDCs). In tissue, immunohistochemistry can identify LCs $\left(\mathrm{CD} 1 \mathrm{a}^{+}\right.$, Langerin $\left.^{+}\right)$and mDCs $\left(\mathrm{CD} 83^{+}\right)$, but other DC phenotypes are not detected reliably in paraffin wax-embedded material. Demedts and colleagues applied an antibody directed against Langerin and thus provide interesting data on the increased numbers of LCs, an important subset of DCs, but their article does not address changes in the numbers of mDCs. Moreover, the LCs that stained positively for Langerin were most likely immature, as Langerin expression is down-regulated upon maturation.

Interestingly, cigarette smoke has been shown in vitro to suppress DC maturation (3), and incubation of LPS-matured DCs with sputum from patients with COPD decreases expression of costimulatory molecules on $\mathrm{mDCs}$ and inhibits $\mathrm{DC}$ maturation (4). Thus, DC maturation is likely attenuated in healthy smokers and in smokers with obstructive airway disease. The reciprocal of this may be an increase in the numbers of iDCs reported by Demedts and coworkers (1).

Finally, decreased DC maturation may shift the $\mathrm{CD} 4^{+} / \mathrm{CD} 8^{+} \mathrm{T}-$ cell ratio in the lungs toward $\mathrm{CD}^{+}$cells, as the time required for DCs to launch the proliferative program for $\mathrm{CD} 8^{+}$is less than that required for $\mathrm{CD}^{+}$cells (5). As the proliferative capacity of $\mathrm{CD}^{+}$cells is greater than that of $\mathrm{CD}^{+}$cells, the latter are more susceptible to the effects of reduced DC maturation (6). The hypothesis that cigarette smoke-induced reduction of DC maturation is associated with development of the "abnormal" or exaggerated inflammation associated with smokers and COPD, or to resistance of asthmatic smokers to steroid therapy, should be tested.

Conflict of Interest Statement: M.T. has no financial relationship with a commercial entity that has an interest in the subject of this manuscript. P.K.J. has been reimbursed by GlaxoSmithKline (GSK), AstraZeneca, and Merck, Sharp \& Dohme (Merck) for attending several conferences and has participated as a paid speaker in scientific meetings or courses organized and financed by GSK, AstraZeneca, Merck, and Boehringer Ingelheim; he has served as a consultant to GSK and Novartis, and in the last 3 years has held research grants from GSK, Merck, and AstraZeneca.

\section{References}

1. Demedts IK, Bracke KR, Pottelberge GV, Testelmans D, Verleden GM, Vermassen FE, Joos GF, Brusselle GG. Accumulation of dendritic cells and increased CCL20 levels in the airways of patients with chronic obstructive pulmonary disease. Am J Respir Crit Care Med 2007;175: 998-1005.

2. Tsoumakidou M, Elston W, Zhu J, Wang Z, Gamble E, Siafakas NM, Barnes NC, Jeffery PK. Cigarette smoking alters bronchial mucosal immunity in asthma. Am J Respir Crit Care Med 2007;175:919-925.

3. Vassallo R, Tamada K, Lau JS, Kroening PR, Chen L. Cigarette smoke extract suppresses human dendritic cell function leading to preferential induction of Th-2 priming. J Immunol 2005;175:2684-2691.

4. Roghanian A, Drost EM, MacNee W, Howie SE, Sallenave J. Inflammatory lung secretions inhibit dendritic cell maturation and function via neutrophil elastase. Am J Respir Crit Care Med 2006;174:1189-1198.

5. van Stipdonk MJ, Hardenberg G, Bijker MS, Lemmens EE, Droin NM, Green DR, Schoenberger SP. Dynamic programming of CD8+ T lymphocyte responses. Nat Immunol 2003;4:361-365.

6. Seder RA, Ahmed R. Similarities and differences in CD4+ and CD8+ effector and memory T cell generation. Nat Immunol 2003;4:835-842.

\section{From the Authors:}

We read with great interest the letter by Tsoumakidou and Jeffery regarding our article on the accumulation of dendritic cells (DCs) in the small airways of patients with chronic obstructive pulmonary disease (COPD) (1). They suggest that DC maturation is attenuated in healthy smokers and in smokers with obstructive airway disease. This hypothesis surely deserves further attention, but our data do not allow confirmation of this statement.

When comparing data from different studies, several methodological issues need to be kept in mind. First, the site of sampling might differ. Findings in large airways are not necessarily the same as in small airways. Second, the study population might be quite different in one study compared with another. The effects of cigarette smoke on DC maturation might be different in asthmatics when compared with patients with COPD or healthy controls. Third, differences in the use of markers to study DCs are of great importance. Both CD1a and Langerin are markers for immature DCs. In the study by Tsoumakidou and colleagues, there was no effect of cigarette smoking on the number of CD1a ${ }^{+}$DCs in bronchial epithelium (2), findings that are consistent with our data obtained in patients with COPD. CD83 is often used as a marker for mature DCs, but several groups reported the expression of CD83 on B lymphocytes and Langerhans' cells (3). Immunohistochemical studies using CD83 as a single marker to identify mature DCs should be interpreted with caution. CD208 may be a better marker to study mature DCs by immunohistochemistry (4).

There are currently no data that allow a conclusion on the effect of cigarette smoke on DC maturation in small airways of patients with COPD. Future cross-sectional studies should be undertaken to investigate the effect of cigarette smoking on both immature and mature DC numbers in healthy controls, patients with COPD, and asthmatics. The subjects in these studies should be carefully matched regarding age, severity of disease, site of sampling, and treatment. Finally, it would be especially useful to start longitudinal studies in which the number of DCs could be evaluated before and after quitting smoking. While this is not possible on tissue sections from surgical specimens, it should be possible to perform these studies using serial bronchial biopsies. 
Conflict of Interest Statement: None of the authors has a financial relationship with a commercial entity that has an interest in the subject of this manuscript.
Ingel K. Demedts
GuY F. Joos
GuY G. Brusselle
Ghent University Hospital
Ghent, Belgium

\section{References}

1. Demedts IK, Bracke KR, Pottelberge GV, Testelmans D, Verleden GM, Vermassen FE, Joos GF, Brusselle GG. Accumulation of dendritic cells and increased CCL20 levels in the airways of patients with chronic obstructive pulmonary disease. Am J Respir Crit Care Med 2007;175: 998-1005.

2. Tsoumakidou M, Elston W, Zhu J, Wang Z, Gamble E, Siafakas NM, Barnes NC, Jeffery PK. Cigarette smoking alters bronchial mucosal immunity in asthma. Am J Respir Crit Care Med 2007;175:919-925.

3. Zhou LJ, Schwarting R, Smith HM, Tedder TF. A novel cell-surface molecule expressed by human interdigitating reticulum cells, Langerhans cells, and activated lymphocytes is a new member of the Ig superfamily. J Immunol 1992;149:735-742.

4. de Saint-Vis B, Vincent J, Vandenabeele S, Vanbervliet B, Pin JJ, itYahia S, Patel S, Mattei MG, Banchereau J, Zurawski S, et al. A novel lysosome-associated membrane glycoprotein, DC-LAMP, induced upon DC maturation, is transiently expressed in MHC class II compartment. Immunity 1998;9:325-336.

\section{Is Giant Cell Interstitial Pneumonitis Synonymous with Hard Metal Lung Disease?}

\section{To the Editor:}

The large case series of hard metal disease described by Moriyami and colleagues in their article provides an important source of clinico-pathologic data pertaining to a rare, but important interstitial lung disease (1). The implications of the authors' reported observations from an immunological perspective were highlighted in the erudite editorial by Nemery and Abraham in the same issue (2). There were, however, important epidemiologic inferences to be drawn from this report that are also worthy of comment.

The authors used electron probe microanalysis to assess the elemental content in lung biopsy material from 85 patients with interstitial lung disease "suspected to be of environmental or occupational origin." Although 23 of the 85 biopsies studied (27\%) manifest pathologic findings of giant cell interstitial pneumonitis (GIP), the basis for study inclusion (i.e., etiological suspicion of an occupational disease) is not elucidated and may very well have included such histology as one criterion. This would be a reasonable approach, given that GIP has come to be treated as a pathologic finding wholly attributable to hard metal or related cobalt-containing exposures. Indeed, this was the stated rationale for "delisting" GIP as an "idiopathic" pneumonia in the 2002 American Thoracic Society/European Respiratory Society (ATS/ERS) statement on the classification of idiopathic interstitial pneumonias (3).

In that light, the further epidemiologic observation of Moriyama and colleagues is all the more intriguing: of the 23 patients with GIP, $2(8.7 \%)$ had no occupational history of exposure (one was a schoolteacher) and no tungsten or cobalt detectable by probe analysis. A recent case report from India of an office sweeper with GIP but no suspect history also raises the question as to whether sporadic cases of this condition occur that cannot be equated with hard metal lung disease (4). There certainly have been cases of GIP in which an occupational history was atypical, but where metals analysis indicated likely occult cobalt exposure (5). Conversely, a thorough metal analysis can be negative, but nonetheless the occupational or environmental history may be highly suspect, as in the case of a 15-year-old with GIP both of whose parents had occupational exposure to hard metal (6). Nemery and Abraham argue cogently that we need to fill in our knowledge gaps about hard metal lung disease (2). Until we do so, it may have been premature to banish GIP from the schema of "idiopathic interstitial pneumonias," as was done in the ATS/ ERS statement (3).

Conflict of Interest Statement: P.D.B. has no financial relationship with a commercial entity that has an interest in the subject of this manuscript.

$$
\begin{aligned}
& \text { Paul D. Blanc } \\
& \text { University of California, San Francisco } \\
& \text { San Francisco, California }
\end{aligned}
$$

\section{References}

1. Moriyama H, Kobayashi M, Takada T, Shimizu T, Terada M, Narita J-I, Maruyuma M, Watanabe K, Suzuki E, Gejyo F. Two-dimensional analysis of elements and mononuclear cells in hard metal lung disease. Am J Respir Crit Care Med 2007;176:70-77.

2. Nemery B, Abraham JL. Hard metal lung disease: still hard to understand. Am J Respir Crit Care Med 2007;176:2-3.

3. American Thoracic Society. American Thoracic Society/European Respiratory Society international multidisciplinary consensus classification of the idiopathic interstitial pneumonias. Am J Respir Crit Care Med 2002;165:277-304.

4. Menon B, Sharma A, Kripalani J, Jain S. Giant cell interstitial pneumonia in a 60-year-old female without hard metal disease. Respiration (Herrlisheim) 2006;73:833-835.

5. Choi JW, Lee KS, Chung MP, Han J, Chung MJ, Park JS. Giant cell interstitial pneumonia: high-resolution CT and pathologic findings in four adult patients. Am J Radiol 2005;184:268-272.

6. Kakugawa T, Mukae H, Nagata T, Ishii H, Kaida H, Hayashi T, Suematsu T, Kadota J-I, Kohno S. Giant cell interstitial pneumonia in a 15 -yearold boy. Intern Med 2002;41:1007-1012.

\section{From the Authors:}

We appreciate Dr. Blanc's comments on our article (1). We screened 85 biopsies; tungsten was detected in surgical biopsies from 17 patients and in transbronchial biopsies (TBBs) from three additional patients. Three TBBs were excluded from our study because the pathologists could not make an accurate pathologic diagnosis due to the small size of the TBB. A TBB from an office sweeper without an exposure history was pathologically diagnosed as giant cell interstitial pneumonia (GIP) (2), but we did not include TBB cases. Even though pathologists find giant cells when screening biopsies, they should be careful in making a final diagnosis of GIP, because giant cells are found in other diseases such as viral pneumonia, especially pneumonia due to measles, and sarcoidosis.

We applied an improved technique for element analysis of tissue sections using an electron probe microanalyzer (EPMA) with a wavelength dispersive spectrometer (WDS) (1). This technique has about 10 times higher sensitivity than EPMA with an energy dispersive spectrometer and enabled us to detect tungsten in lung tissue in which the element was not found by the other method (unpublished data; Reference 3). We found two biopsies exhibiting features of GIP with neither tungsten nor cobalt detected in the lung tissue by EPMA-WDS nor a working history in the hard metal industry. Finally, 2 (10.5\%) of 19 surgical lung biopsies were thought to be "idiopathic" GIP in our case series.

In addition to EPMA, atomic absorption spectrometry, plasma optical emission mass spectrometry, and ionic coupled plasma emission spectrometry have been used to detect tungsten and cobalt in lung tissue $(2,4,5)$. All of these techniques are methods to detect elements in dissolved tissue solution. Using these methods, one cannot see the relationship between elements and the characteristic lesions of GIP, including centrilobular fibrosis and giant cell accumulation within alveolar space; thus, some GIP diagnosed as hard metal lung disease might actually be "idiopathic" GIP. 